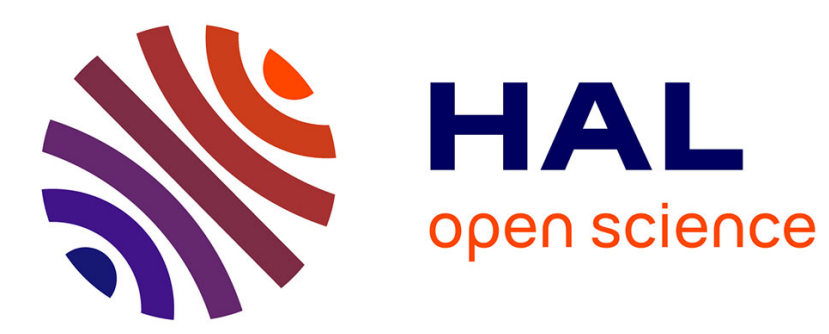

\title{
Spectrométrie de neutrons rapides
}

G. Deconninck, J.P. Meulders

\section{To cite this version:}

G. Deconninck, J.P. Meulders. Spectrométrie de neutrons rapides. Revue de Physique Appliquée, 1969, 4 (2), pp.243-244. 10.1051/rphysap:0196900402024300 . jpa-00243242

\section{HAL Id: jpa-00243242 https://hal.science/jpa-00243242}

Submitted on 1 Jan 1969

HAL is a multi-disciplinary open access archive for the deposit and dissemination of scientific research documents, whether they are published or not. The documents may come from teaching and research institutions in France or abroad, or from public or private research centers.
L'archive ouverte pluridisciplinaire HAL, est destinée au dépôt et à la diffusion de documents scientifiques de niveau recherche, publiés ou non, émanant des établissements d'enseignement et de recherche français ou étrangers, des laboratoires publics ou privés. 


\title{
SPEGTROMÉTRIE DE NEUTRONS RAPIDES
}

\author{
G. DEGONNINCK* et J. P. MEULDERS**, \\ Centre de Physique Nucléaire, Heverlé-Louvain, \\ * Facultés Universitaires de Namur et Université de Louvain, **Université de Louvain.
}

\begin{abstract}
Résumé. - Une méthode de dépouillement de spectres de neutrons obtenus par la méthode du temps-de-vol est décrite. La séparation des groupes de neutrons ainsi que la correction d'efficience sont obtenues par méthodes numériques.
\end{abstract}

Abstract. - A data processing method is presented which allows the determination of the neutron component in time of flight spectra. Separation of neutrons as well as efficiency corrections are obtained by numerical methods.

Dans l'identification des spectres de neutrons obtenus à partir de la méthode du temps-de-vol, on se heurte à deux difficultés : la séparation des différentes composantes et la détermination de l'efficience (qui est variable avec l'énergie). Dans ce qui suit, nous décrivons les méthodes utilisées pour résoudre ces deux problèmes dans le cas où deux composantes de neutrons

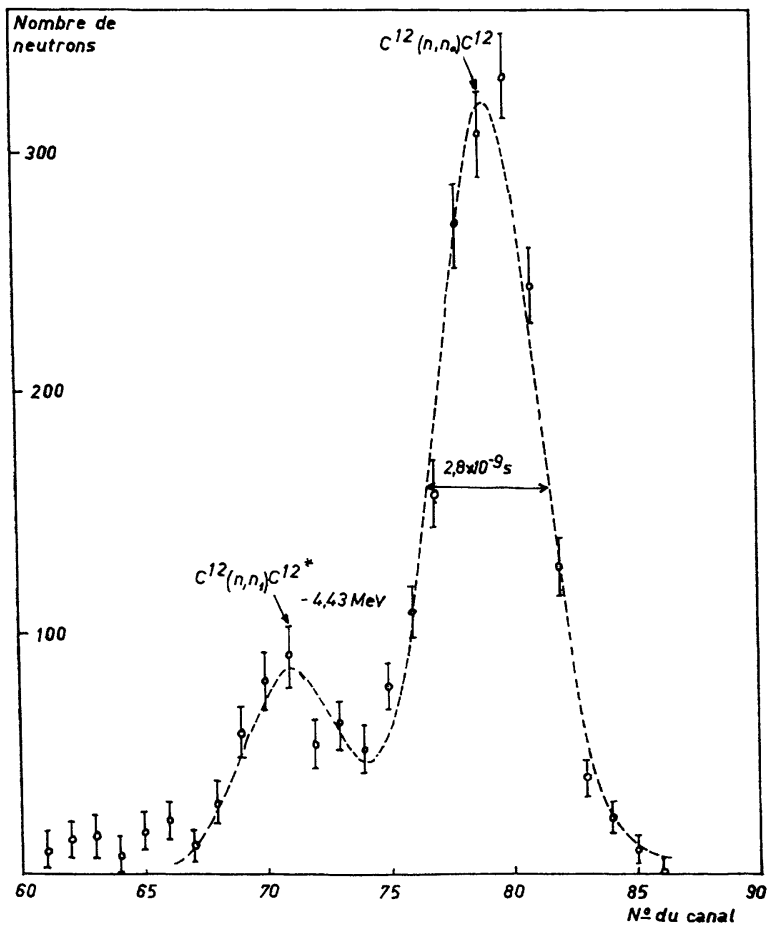

Fig. 1. - Spectre de temps-de-vol des neutrons de $19,9 \mathrm{MeV}$, diffusés par ${ }^{12} \mathrm{C}$ à $40^{\circ}: \stackrel{!}{\circ}$, nombre de neutrons enregistrés ; - - -, calcul à l'aide d'une somme de deux gaussiennes. sont présentes. La méthode s'étend immédiatement aux cas où les spectres comprennent un plus grand nombre de groupes de neutrons.

La réaction $\mathrm{T}(\mathrm{d}, \mathrm{n}){ }^{4} \mathrm{He}$ a été utilisée à l'accélérateur Van de Graaff de 4,4 MeV pour l'étude, par la méthode de temps-de-vol, de la diffusion élastique et inélastique de neutrons rapides sur ${ }^{12} \mathrm{G}$ aux énergies comprises entre $17 \mathrm{MeV}$ et $20 \mathrm{MeV}$. Les spectres de temps-de-vol présentent deux pics, dus à la diffusion élastique et inélastique sur le premier niveau excité ( fig. 1) ; ces pics sont trop voisins pour être séparés simplement. Nous avons mis au point un programme de dépouillement, permettant de tracer à travers les points expérimentaux la somme incohérente de deux gaussiennes. La courbe calculée peut s'écrire :

$$
y=\frac{A_{0}}{s \sqrt{2 \pi}} e^{-\left[\frac{x-(\mu-\delta)}{\sqrt{2} s}\right]^{2}}+\frac{B_{0}}{s \sqrt{2 \pi}} e^{-\left[\frac{x-\mu}{\sqrt{2} s}\right]^{2}}
$$

où $A_{0}$ et $B_{0}$ représentent les amplitudes des deux pics; $\mu$, le canal positionnant le maximum; la distance $(\delta)$ entre les deux maximums est fixée par l'énergie des deux groupes de neutrons; $s$, la variance des distributions, la même dans les deux cas, car elle est une mesure de la résolution totale de l'expérience.

Après la minimalisation du $\chi^{2}$, obtenue par recherche automatique sur les paramètres variables $\mu$ et $s$, l'on obtient les amplitudes des gaussiennes cherchées (courbe pointillée de la figure 1).

D'autre part, l'efficience relative du détecteur, au scintillateur liquide $5^{\prime \prime} \times 3^{\prime \prime}$, a été mesurée en deux étapes : 1) entre $12,3 \mathrm{MeV}$ et $19,6 \mathrm{MeV}$ par la distribution angulaire des neutrons de la réaction $\mathrm{T}(\mathrm{d}, \mathrm{n})^{4} \mathrm{He}$; 2) entre $8,2 \mathrm{MeV}$ et $16,3 \mathrm{MeV}$ par la diffusion des neutrons sur les protons d'un cylindre en polyéthylène $(\mathrm{CH})_{\mathrm{n}}$. Dans cette deuxième réaction, il faut d'abord évaluer la contribution du pic ${ }^{12} \mathrm{C}(\mathrm{n}, \mathrm{n}){ }^{12} \mathrm{C}$ aux 
angles où il se distingue du pic ${ }^{1} \mathrm{H}(\mathrm{n}, \mathrm{n})^{1} \mathrm{H}$ (c'est-à-dire de $45^{\circ}$ à $55^{\circ}$ ); ceci peut se faire par la méthode des gaussiennes, décrite plus haut.

Un programme de calcul d'absorption et de diffusion

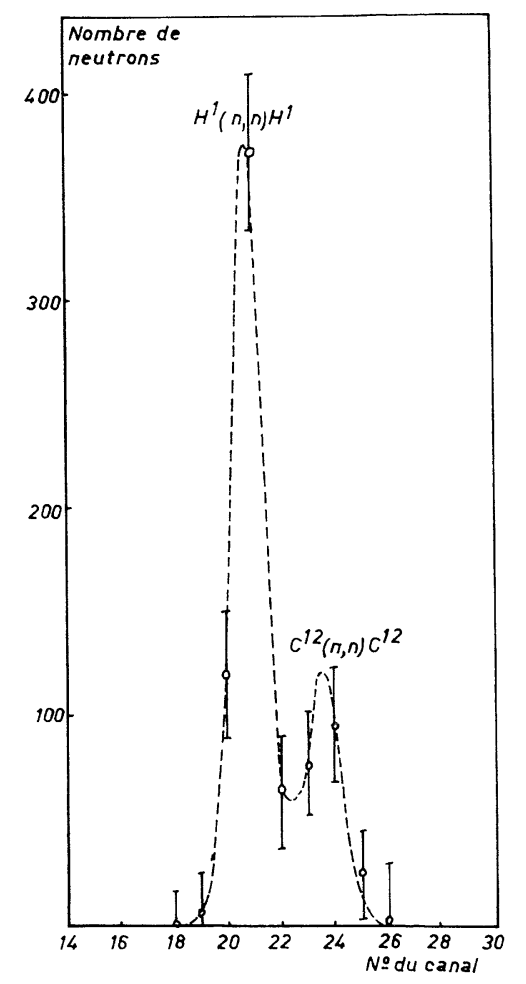

FIG. 2. - Spectre de diffusion de neutrons de $20 \mathrm{MeV}$ sur le polyéthylène à $50^{\circ}$. simple et double permet ensuite d'évaluer, par la méthode de Monte-Carlo employée dans le cas d'une géométrie cylindrique, la diffusion des neutrons $\operatorname{sur}^{12} \mathrm{C}$, afin de la soustraire de celle des neutrons sur les protons, aux angles où les deux pics se recouvrent (c'est-à-dire de $25^{\circ}$ à $40^{\circ}$ ). La même méthode permet ensuite d'évaluer la diffusion des neutrons dans un milieu composé de polyéthylène. Enfin, la même méthode permet d'obtenir la section efficace différentielle de diffusion élastique et inélastique des neutrons sur ${ }^{12} \mathrm{C}$ de manière absolue à partir des sections efficaces, mesurées dans le cas d'une géométrie cylindrique. L'évaluation de la contribution de diffusion double de neutrons est en excellent accord, dans le cas de ${ }^{12} \mathrm{C}$, avec les résultats de Cox [1], ce qui permet d'employer ses résultats pour l'évaluation de la diffusion triple. D'autre part, nous avons également traité le cas général, qui consiste à considérer la direction des trajectoires des neutrons, incidents à l'échantillon et émergents de l'échantillon, de manière tout à fait quelconque (et non horizontale comme on le fait généralement). Nous avons montré [2] que cette généralisation peut donner lieu à un effet important, dès qu'une des distances source de neutrons-échantillon ou échantillon-détecteur devient comparable aux dimensions de l'échantillon; la valeur absolue de la section efficace différentielle se modifie principalement aux extrêmes de la distribution angulaire.

Le cas de la diffusion de neutrons dans une géométrie annulaire a également été traité par cette méthode [3].

Nous remercions la direction et le personnel du Gentre de Calcul numérique de Louvain pour les facilités d'accès à l'ordinateur IBM 360/40, ainsi que l'I.I.S.N. qui finance ces recherches.

\section{BIBLIOGRAPHIE}

[1] Cox (S. A.), Nucl. Instr. Methods, 1967, 56, 245.

[2] Meulders (J. P.), Ann. Soc. Sci. Bruxelles, 1968, $82,307$.

[3] Meulders (J. P.), Monseu (Ph.) et Deconninck (G.), Ann. Soc. Sci. Bruxelles, 1967, 81, 149. 